PHYSICAL REVIEW D 96, 129902(E) (2017)

\title{
Erratum: Electron exchange and polarization effects on electron captures and neutron emissions by nuclei in white dwarfs and neutron stars [Phys. Rev. D 93, 063001 (2016)]
}

\author{
N. Chamel and A. F. Fantina \\ (Received 21 November 2017; published 20 December 2017)
}

DOI: 10.1103/PhysRevD.96.129902

The Equation (17) for the plasma temperature should read

$$
T_{p}=\frac{m_{e} c^{2}}{k_{\mathrm{B}}} \sqrt{\frac{4}{3 \pi} \frac{m_{e}}{M^{\prime}}} \sqrt{\alpha Z}\left(\frac{\mu_{e}^{\beta n}}{m_{e} c^{2} \Delta Z}\right)^{3 / 2} \approx 7.73 \times 10^{6} \sqrt{\frac{Z}{A}}\left(\frac{\mu_{e}^{\beta n}}{m_{e} c^{2} \Delta Z}\right)^{3 / 2} \mathrm{~K}
$$

In Section IIIB, the plasma parameter $\Gamma_{p}$ was incorrectly estimated from Eq. (21) with $\mu_{e} \approx \mu_{e}^{\text {drip }} / Z$ instead of $\mu_{e} \approx \mu_{e}^{\text {drip }}$. Revised values for $\Gamma_{p}$ are of the order of $3 \times 10^{2}$. Since $\Gamma_{p} \gg 1$, the electron polarization formulas of Potekhin and Chabrier [1] can still be very well approximated by Eqs. (6) and (8).

The values for the characteristic temperatures given in Table II contained errors. Corrected values are indicated in Table I. The validity of our treatment of electron polarization at finite temperatures is thus still limited by the plasma temperature, but whose estimates are now much higher, namely $T_{p} \sim 10^{9} \mathrm{~K}$. This corresponds to an effective surface temperature (as seen by an observer at infinity) between $10^{6.6} \mathrm{~K}$ and $10^{6.8} \mathrm{~K}$ (see Fig. 7 of Ref. [2]). Depending on the cooling scenario, our model is thus applicable to neutron stars whose estimated age ranges from a few months up to several million years [3]. Therefore, our model remains suitable for neutron stars younger than previously found.

At the temperatures $T<T_{p} \sim 10^{9} \mathrm{~K}$ considered here, thermal effects are not expected to play a role, since thermal corrections are exponentially suppressed by shell and pairing effects. Indeed, as shown in Ref. [4], the composition of neutron-star crusts at the neutron-drip transition remains unchanged up to temperatures of the order of $10^{9} \mathrm{~K}$.

TABLE I. Characteristic temperatures at the neutron-drip transition in the crust of nonaccreting neutron stars, as predicted by different nuclear mass models: electron Fermi temperature $T_{\mathrm{Fe}}$, crystallization temperature $T_{m}$, and plasma temperature $T_{p}$.

\begin{tabular}{lccc}
\hline \hline & $T_{\mathrm{Fe}}(\mathrm{K})$ & $T_{m}(\mathrm{~K})$ & $T_{p}(\mathrm{~K})$ \\
\hline HFB-22 & $2.9 \times 10^{11}\left(2.9 \times 10^{11}\right)$ & $2.7 \times 10^{9}\left(2.5 \times 10^{9}\right)$ & $1.4 \times 10^{9}\left(1.4 \times 10^{9}\right)$ \\
HFB-24 & $2.9 \times 10^{11}$ & $2.7 \times 10^{9}$ & $1.4 \times 10^{9}$ \\
HFB-27 & $2.9 \times 10^{11}$ & $2.7 \times 10^{9}$ & $1.4 \times 10^{9}$ \\
D1M & $2.9 \times 10^{11}$ & $2.7 \times 10^{9}$ & $1.5 \times 10^{9}$ \\
DZ & $2.9 \times 10^{11}$ & $2.5 \times 10^{9}$ & $1.4 \times 10^{9}$ \\
\hline \hline
\end{tabular}

[1] A. Y. Potekhin and G. Chabrier, Phys. Rev. E 62, 8554 (2000).

[2] D. Page, U. Geppert, and F. Weber, Nucl. Phys. A777, 497 (2006).

[3] O. Y. Gnedin, D. G. Yakovlev, and A. Y. Potekhin, Mon. Not. R. Astron. Soc. 324, 725 (2001).

[4] M. Onsi, A. K. Dutta, H. Chatri, S. Goriely, N. Chamel, and J. M. Pearson, Phys. Rev. C 77, 065805 (2008). 